\title{
ADDITION OF AN IDENTITY TO AN ORDERED BANACH SPACE
}

\author{
DEREK W. ROBINSON and SADAYUKI YAMAMURO
}

(Received 11 June 1982)

Communicated by W. Moran

\begin{abstract}
Given an ordered Banach space $\mathscr{B}$ equipped with an order-norm we construct a larger space $\tilde{B}$ with an order-norm and order-identity such that $\mathscr{B}$ is isometrically order-isomorphic to a Banach subspace of $\tilde{B}$. We also discuss the extension of positive operators from $\mathscr{B}$ to $\tilde{B}$.
\end{abstract}

1980 Mathematics subject classification (Amer. Math. Soc.): 46 B 20.

\section{Introduction}

The addition of an identity is a standard technique in the theory of $C^{*}$-algebras. In this note we examine a similar construction for ordered Banach spaces. Given a Banach space $\mathscr{B}$ ordered by a positive cone $\mathscr{B}_{+}$we construct a larger space $\tilde{\mathscr{B}}=(\mathscr{B}, \mathbf{R})$ ordered by a positive cone $\tilde{\mathscr{B}}_{+}$and equipped with an order-norm $\|\cdot\|_{+}$which ensures that $e=(0,1)$ is an (order-) identity of $\tilde{\mathscr{B}}$, that is, $e$ is maximal in the unit ball of $\left(\tilde{\mathscr{B}}, \tilde{\mathscr{B}}_{+},\|\cdot\|_{+}\right)$. The embeddings $(\mathscr{\mathscr { B }}, 0) \subset \tilde{\mathscr{B}}$ and $\left(\tilde{T}_{+}, 0\right) \subset \tilde{\mathscr{B}}_{+}$are however isometric order isomorphisms if, and only if, the norm $\|\cdot\|$ on $\mathscr{B}$ coincides with the order-norm. This is the case for $C^{*}$-algebras.

\section{The order-norm and order-identity}

Let $(\mathscr{R},\|\cdot\|)$ be a real Banach space ordered by a positive cone $\mathscr{B}_{+}$, that is, $\mathscr{B}_{+}$ is a norm-closed convex cone in $\mathscr{G}$ satisfying

$$
\mathscr{B}_{+} \cap-B_{+}=\{0\}
$$

C 1983 Australian Mathematical Society 0263-6115/83 \$A2.00+0.00 
and the relation $a \geqslant b$ is defined by $a-b \in \mathscr{B}_{+}$. We define the order half-norm $N_{+}$, as a positive function over $\mathscr{B}$, by

$$
N_{+}(a)=\inf \left\{\lambda \geqslant 0 ; a \leqslant \lambda u \text { for some } u \in \mathscr{B}_{1}\right\}
$$

and the order-norm $\|\cdot\|_{+}$by

$$
\begin{aligned}
\|a\|_{+} & =N_{+}(a) \vee N_{+}(-a) \\
& =\inf \left\{\lambda \geqslant 0 ;-\lambda u \leqslant a \leqslant \lambda v \text { for some } u, v \in \mathscr{B}_{1}\right\},
\end{aligned}
$$

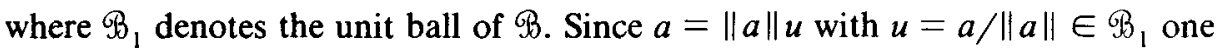
has $N_{+}(a) \leqslant\|a\|$ and hence

$$
\|a\|_{+} \leqslant\|a\|
$$

for all $a \in \mathscr{B}$. But in general the two norms are inequivalent. Before further comparison of the norms we mention an alternative characterization of the order half-norm and hence the order-norm.

LEMMA 1.1. Let $\mathscr{B}$ be a Banach space with positive cone $\mathscr{G}_{+}$and order half-norm $N_{+}$. It follows that

$$
N_{+}(a)=\inf \left\{\|a+b\| ; b \in \mathscr{B}_{+}\right\} \text {. }
$$

Proof. If $a \leqslant \lambda u$ for some $u \in \mathscr{B}_{1}$ then $\lambda u=a+b$ for some $b \in \mathscr{B}_{+}$and $|\lambda| \geqslant\|a+b\|$. Thus

$$
N_{+}(a) \geqslant \inf \left\{\|a+b\| ; b \in \mathscr{B}_{+}\right\} .
$$

But the converse inequality follows because one has $a \leqslant\|a+b\| u$, with $u=$ $(a+b) /\|a+b\| \in \mathscr{B}_{1}$, for each $b \in \mathscr{G}_{+}$.

REMARK. The order half-norm is implicit in the work of Grosberg and Krein [6] and occurs explicitly in the work of Kadison [7]. It is basic to the introduction of the order-norm on an 'order-unit' space [1], [3]. More recently Arendt, Chernoff and Kato [2] defined the order half-norm by the criterion of Lemma 1 and called it the canonical half-norm. Note that $N_{+}$is determined by $\mathscr{B}_{+}$and conversely

$$
\mathscr{B}_{+}=\left\{a ; N_{+}(-a)=0\right\} \text {. }
$$

Equivalence of the norm and order-norm is basically a property of the positive cone $\mathscr{B}_{+}$. Krein [8] was the first to introduce the appropriate notion of a normal cone.

The cone $\mathscr{B}_{+}$is defined to be $\alpha$-normal if there is an $\alpha \geqslant 1$ such that $a \leqslant b \leqslant c$ always implies

$$
\|b\| \leqslant \alpha(\|a\| \vee\|c\|) \text {. }
$$

There are various alternative definitions of normality (see, for example, [9] Chapter 2). In particular it can be characterized in terms of positive functions. 
An element of the dual $\mathscr{B}^{*}$ of $\mathscr{B}$ is defined to be positive, $f \geqslant 0$, if

$$
f(a) \geqslant 0
$$

for all $a \in \mathscr{B}_{+}$. The set of positive functionals $f \in \mathscr{B}^{*}$ forms a norm-closed convex cone $\mathscr{B}_{+}^{*}$ which is called the dual cone. The cone is said to be $\alpha$-generated if each $f \in \mathscr{B}^{*}$ has a decomposition $f=f_{+}-f_{-}$with $f_{ \pm} \in \mathscr{B}_{+}^{*}$ and

$$
\alpha\|f\| \geqslant\left\|f_{+}\right\|+\left\|f_{-}\right\| .
$$

Note that if $\mathscr{B}_{+}^{*}$ is 1 -generated then it follows from the triangle inequality that each $f \in \mathscr{B}^{*}$ has a Jordan decomposition, that is, $f=f_{+}-f_{-}$with $f_{ \pm} \in \mathscr{B}_{+}^{*}$ and

$$
\|f\|=\left\|f_{+}\right\|+\left\|f_{-}\right\| \text {. }
$$

The following proposition gives criteria for equivalence, and equality, of the norm $\|\cdot\|$ and order-norm $\|\cdot\|_{+}$on $\mathscr{B}$. In particular it restates Grosberg and Krein's result on the equivalence of $\alpha$-normality of $\mathscr{B}_{+}$and $\alpha$-generation of $\mathscr{B}_{+}^{*}$.

Proposition 1.2. Let $\left(\mathscr{B}, \mathscr{B}_{+},\|\cdot\|\right)$ be an ordered Banach space with corresponding order-norm $\|\cdot\|$. The following conditions are equivalent:

1. $\|\cdot\|$ and $\|\cdot\|_{+}$are equivalent norms,

2. $\mathscr{B}_{+}$is $\alpha$-normal, for $\alpha \geqslant 1$,

3. $9_{+}^{*}$ is $\alpha$-generated, for $\alpha \geqslant 1$.

Moreover the following conditions are equivalent:

$1^{\prime} .\|\cdot\|=\|\cdot\|_{+}$,

$2^{\prime} \cdot 9_{3}$ is 1-normal,

3'. each $f \in \Re^{*}$ has a Jordan decomposition.

Proof. The equivalence of Conditions 2 and 3, and hence of Conditions $2^{\prime}$ and 3', was established by Grosberg and Krein [6] (see also [3] Chapter 2). We will prove $1 \Leftrightarrow 2$ and simultaneously $\mathrm{l}^{\prime} \Leftrightarrow 2^{\prime}$.

$1 \Rightarrow 2$. Assume $\|b\| \leqslant \alpha\|b\|_{+}$for all $b \in \mathfrak{B}$. But $a \leqslant b \leqslant c$ implies directly that $\|b\|_{+} \leqslant\|a\| \vee\|c\|$ and hence

$$
\|b\| \leqslant \alpha(\|a\| \vee\|c\|) .
$$

Setting $\alpha=1$ one deduces that $1^{\prime} \Rightarrow 2^{\prime}$.

$2 \Rightarrow 1$. Since $\mathscr{B}_{+}$is $\alpha$-normal the relations $-\lambda u \leqslant a \leqslant \lambda v$ with $u, v \in \mathscr{B}_{1}$ imply that $\|a\| \leqslant \alpha \lambda$ and hence

$$
\|a\| \leqslant \alpha\|a\|_{+} .
$$

But $\|a\|_{+} \leqslant\|a\|$ and hence the norms are equivalent.

Again setting $\alpha=1$ one concludes that $2^{\prime} \Rightarrow 1^{\prime}$.

EXAMPLE 1.3. If $\mathscr{B}_{+}=\{0\}$ then $\|\cdot\|_{+}=\|\cdot\|$ and $\mathscr{B}_{+}^{*}=\mathscr{G}^{*}$. 
EXAMPLE 1.4. If $\mathscr{B}$ is the hermitian part of a $C^{*}$-algebra $\mathfrak{A}$ ordered by the positive elements $\mathfrak{A}_{+}$of the algebra then $\|\cdot\|=\|\cdot\|_{+}$because each $f \in \mathfrak{A}^{*}$ has a Jordan decomposition [5], that is, the $C^{*}$-norm and order-norm coincide. This equality of norms can also be established by direct calculation.

EXAMPLE 1.5. Let $\mathscr{B}$ be an order complete Banach lattice (see, for example, [10]). Then $\|\cdot\|=\|\cdot\|_{+}$if, and only if, $\mathscr{B}$ is an $A M$-space, that is, $\|a \vee b\|=$ $\|a\| \vee\|b\|$ for all $a, b \in \mathscr{B}_{+}$. This is established by first remarking that the dual of an $A M$-space is an $A L$-space [10] and each element of an $A L$-space has a Jordan decomposition, that is, Condition 3 of Proposition 1.2 is valid. Conversely each $a \in \mathscr{B}$ has a canonical decomposition [10] $a=a_{+}-a_{-}$with $a_{ \pm} \in \mathscr{B}_{+}$and $a_{+} \wedge a_{-}=0$. But $N_{+}(a)=\left\|a_{+}\right\|[2]$ and hence if $\|\cdot\|=\|\cdot\|_{+}$then

$$
\|a\|=\|a\|_{+}=\left\|a_{+}\right\| \vee\left\|a_{-}\right\| .
$$

Since $a_{+} \wedge a_{-}=0$ this is also equivalent to

$$
\|a \vee b\|=\|a\| \vee\|b\|
$$

for all $a, b \in \mathscr{B}_{+}$with $a \wedge b=0$. To remove this last restriction take $a, b \in \mathscr{B}_{+}$ and define $a_{1}, b_{1} \in \mathscr{B}_{+}$by

$$
a_{1}=a \vee b-b, \quad b_{1}=a \vee b-a .
$$

One then readily checks that $a_{1} \wedge b_{1}=0$.

Next define $\mathcal{Q}$ and $\mathcal{Q}^{\perp}$ by

$$
\mathbb{Q}=\left\{a ; a \wedge b_{1}=0\right\}, \quad \mathbb{Q}^{\perp}=\{b ; b \wedge a=0, a \in \mathbb{Q}\} .
$$

It follows from the assumed order completeness that $\mathscr{B}=\mathscr{Q} \oplus \mathbb{Q}^{\perp}$ ([10] Chapter 2, Theorem 2.10) and the projection $P: \mathscr{B} \mapsto \mathbb{Q}$ is positive and continuous with $\|P\| \leqslant 1$ ([10] Chapter 2, Propositions 2.7 and 5.2). Therefore

$$
P(a \vee b)-P a=P b_{1}=0
$$

because $b_{1} \in \mathbb{Q}^{\perp}$ and

$$
(1-P)(a \vee b)-(1-P) b=(1-P) a_{1}=0
$$

because $a_{1} \in \mathbb{Q}$. Therefore if

$$
a^{\prime}=P(a \vee b), \quad b^{\prime}=(1-P)(a \vee b)
$$

one has $a^{\prime} \wedge b^{\prime}=0$ and $a^{\prime}+b^{\prime}=a^{\prime} \vee b^{\prime}=a \vee b$. Moreover $0 \leqslant a^{\prime}=P a \leqslant a$, $0 \leqslant b^{\prime}=(1-P) b \leqslant b$. Consequently

$$
\|\boldsymbol{a} \vee \boldsymbol{b}\|=\left\|a^{\prime} \vee b^{\prime}\right\|=\left\|a^{\prime}\right\| \vee\left\|b^{\prime}\right\| \leqslant\|a\| \vee\|b\|
$$

where the second step uses $a^{\prime} \wedge b^{\prime}=0$. But $a \vee b \geqslant a, a \vee b \geqslant b$. Hence

$$
\|a \vee b\| \geqslant\|a\| \vee\|b\|
$$

and this establishes that $\mathscr{B}$ is an $A M$-space. 
Next we consider identity elements.

An element $e$ of the ordered Banach space $\left(\mathscr{B}, \mathscr{B}_{+},\|\cdot\|\right)$ is defined to be an (order-) identity, or unit element, if it is maximal, with respect to the order induced by $\mathscr{B}_{+}$, in the unit ball $\mathscr{B}_{1}$. There are alternative characterizations:

PROPOSITION 1.6. Let e be an element of the unit ball of the ordered Banach space $\left(\mathscr{B}, \mathscr{B}_{+},\|\cdot\|\right)$. The following conditions are equivalent:

1. $e$ is an identity of $B$,

2. $\{a ;\|a-e\|<1\} \subset \mathscr{B}_{+}$,

3. $N_{+}=N_{e}$ where $N_{+}$is the order half-norm and

$$
N_{e}(a)=\inf \{\lambda \geqslant 0 ; a \leqslant \lambda e\} .
$$

Hence if $\mathscr{B}$ has an identity $e$ the order-norm is characterized by

$$
\begin{aligned}
\|a\|_{+} & =\inf \left\{\lambda \geqslant 0 ;-\lambda u \leqslant a \leqslant \lambda v ; u, v \in \mathscr{B}_{1} \cap \mathscr{B}_{+}\right\} \\
& =\inf \{\lambda \geqslant 0 ;-\lambda e \leqslant a \leqslant \lambda e\}
\end{aligned}
$$

and, moreover, $e$ is an identity of $\left(\mathscr{B}_{,} \mathscr{B}_{+},\|\cdot\|_{+}\right)$.

Proof. $1 \Rightarrow 3$. Since $e \in \mathscr{B}_{1}$ one has $N_{e} \geqslant N_{+}$. But conversely if $v \in \mathscr{B}_{1}$ then $v \leqslant e$, by maximality, and hence

$$
N_{+}(a)=\inf \left\{\lambda \geqslant 0 ; a \leqslant \lambda v, v \in \mathscr{B}_{1}\right\} \geqslant \inf \{\lambda \geqslant 0 ; a \leqslant \lambda e\}=N_{e}(a) .
$$

Thus $N_{+}=N_{e}$.

$3 \Rightarrow 2$. The order half-norm satisfies $N_{+}(a) \leqslant\|a\|$. Hence if $N_{+}=N_{e}$ then $a \leqslant\|a\| e$ for all $a \in \mathscr{B}$. Consequently the unit ball around $e$ is contained in $\mathscr{B}_{+}$.

$2 \Rightarrow 1$. If $b=e-a /\|a\|(1+\varepsilon)$ then $\|e-b\| \leqslant 1 /(1+\varepsilon)$ and hence $b \geqslant 0$ for $\varepsilon>0$, that is, $a \leqslant\|a\|(1+\varepsilon) e$. But since $\mathscr{B}_{+}$is norm-closed one then has

$$
a \leqslant\|a\| e
$$

and hence $e$ is maximal in $\mathscr{B}_{1}$.

Now it follows from Condition 3 that

$$
\|a\|_{+}=\inf \{\lambda \geqslant 0 ;-\lambda e \leqslant a \leqslant \lambda e\}
$$

and the other characterization results from the fact that $e \in \mathscr{B}_{1} \cap \mathscr{B}_{+}$. Finally (*) implies that $e \geqslant a /\|a\|_{+}$and hence, by maximality, $e$ is an identity of $\left(\mathscr{G}, \Re_{+},\|\cdot\|_{+}\right)$.

Remark. A little care must be taken with the last statement of Proposition 1.6. If $\mathscr{B}_{+}$is not normal with respect to $\|\cdot\|$ the space $\mathscr{B}_{3}$ is not $\|\cdot\|_{+}-$complete, and the cone $\mathscr{B}_{+}$is not $\|\cdot\|_{+}$-closed. But $\mathscr{B}$ can be $\|\cdot\|_{+}$-completed, and $\mathscr{B}_{+}$can be 
$\|\cdot\|_{+}$-closed. The identity $e$ of $(\Re, \Re,\|\cdot\|)$ then remains an identity for the $\|\cdot\|_{+}$-completed space $\left(\overline{\mathscr{B}}, \overline{\mathrm{B}}_{+},\|\cdot\|_{+}\right)$. The order-norm is the smallest norm with this property.

Note that if $\mathscr{B}$ has an identity then it is unique because maximality of both $e_{1}$ and $e_{2}$ in $\mathscr{B}_{1}$ implies $\pm\left(e_{1}-e_{2}\right) \in \mathscr{B}_{+}$and hence $e_{1}=e_{2}$. But not all ordered Banach spaces have an identity $e$. In fact Condition 2 of Proposition 1.6 demonstrates that $e$ is an interior point of $\mathscr{B}_{+}$and in many cases $\mathscr{B}_{+}$has an empty interior.

EXAMPLE 1.7. If $\mathscr{B}$ is the hermitian part of a $C^{*}$-algebra $\mathfrak{A}$ ordered by the positive elements $\mathfrak{A}_{+}$of the algebra then $\mathscr{B}$ has an (order-) identity if, and only if, $\mathfrak{U}$ has an (algebraic-) identity and in this case the two coincide.

EXAMPLE 1.8. If $(\mathscr{B},\|\cdot\|)$ is a Banach lattice then $\mathfrak{B}_{+}$has interior points if, and only if, $\mathscr{B}$ is lattice isomorphic to $C(X)$ for some compact Hausdorff space $X$. (See, for example [4].) Moreover if $u$ is an interior point of $\mathscr{G}_{+}$then each $a \in \mathscr{B}$ can be majorized by a multiple of $u$ and hence one can introduce the norm

$$
\|a\|_{u}=\inf \{\lambda \geqslant 0 ;-\lambda u \leqslant a \leqslant \lambda u\} .
$$

It follows that $\|\cdot\|_{u}$ is equivalent to $\|\cdot\|$ and $\left(\Re,\|\cdot\|_{u}\right)$ is an $A M$-space with identity $u$ (again see [4]).

\section{Addition of an identity}

Next we consider the embedding of an ordered Banach space $\left(\mathscr{B}_{,} \mathscr{B}_{+},\|\cdot\|\right)$ in a larger space $\left(\tilde{\mathscr{B}}, \tilde{\mathscr{B}}_{+},\|\cdot\|_{+}\right)$with an identity $e$ and corresponding order-norm $\|\cdot\|_{+}$. This embedding is an order-isomorphism but is not necessarily isometric. But again we remark that $\mathscr{B}$ can be completed with respect to the order-norm $\|\cdot\|_{+}$, and $\mathscr{B}_{+}$can be closed. The embedding theorem then gives an isometric order-isomorphism of the completed space $\left(\overline{\mathscr{S}}_{\hat{B}}, \overline{\mathscr{B}}_{+},\|\cdot\|_{+}\right)$in the space $\left(\tilde{\mathscr{G}}_{\bar{B}}, \tilde{\mathscr{B}}_{+}\right.$, $\|\cdot\|_{+}$) with identity. For simplicity we will not distinguish between $\mathscr{G}$ and $\overline{\mathscr{B}}, \mathscr{G}_{+}$ and $\overline{\mathscr{B}}_{+}$, in the sequel.

THEOREM 2.1. Let $\left(\mathscr{B}, \mathscr{B}_{+},\|\cdot\|_{+}\right)$be an ordered Banach space equipped with the order norm $\|\cdot\|_{+}$. Consider the space $\tilde{\mathscr{B}}=(\mathscr{B}, \mathbf{R})$ of pairs $(a, t)$ with $a \in \mathscr{B}$, $t \in \mathbf{R}$, with the operations

$$
\lambda(a, t)=(\lambda a, \lambda t), \quad(a, t)+(b, s)=(a+b, s+t),
$$

with the norm

$$
\|(a, t)\|_{+}=\left(N_{+}(a)+t\right) \vee\left(N_{+}(-a)-t\right),
$$


and with the cone

$$
\tilde{\mathscr{G}}_{+}=\left\{(a, t) ; t \geqslant N_{+}(-a)\right\} .
$$

It follows that $\left(\tilde{\mathscr{B}}_{\tilde{B}} \tilde{\mathscr{B}}_{+},\|\cdot\|_{+}\right)$is an ordered Banach space, $\mathscr{B}=(\mathscr{B}, 0)$ is a Banach subspace of $\tilde{\mathscr{B}}, \mathscr{B}_{+}=\left(\mathscr{B}_{+}, 0\right)$ is a positive subcone of $\tilde{\mathscr{B}}_{+}, e=(0,1)$ is an identity of $\left(\tilde{\mathscr{G}}, \tilde{\mathscr{B}}_{+},\|\cdot\|_{+}\right)$and $\|\cdot\|_{+}$is the order-norm on $\tilde{\mathscr{B}}$ defined by $\tilde{\mathscr{B}}_{+}$.

Proof. First consider the space $\tilde{\mathscr{B}}=(\mathscr{B}, \mathbf{R})$ with the norm

$$
\|(a, t)\|=\|a\|_{+}+|t| \text {. }
$$

Since $\mathscr{B}$ is $\|\cdot\|_{+}$-complete $\tilde{\mathscr{B}}$ is automatically $\|\cdot\|$-complete and one can identify $\mathscr{B}=(\mathscr{G}, 0)$ as a Banach subspace. Now since $N_{+}$is a half-norm $\tilde{\mathscr{G}}_{+}$is a proper norm-closed convex cone. For example if $\pm(a, t) \in \tilde{\mathscr{B}}_{+}$then $\pm t \geqslant N_{+}( \pm a)$ and $t=0=N_{+}(a)=N_{+}(-a)$, because $N_{+}$is positive. Hence $a=0=t$. Note that if $a \in \mathscr{B}_{+}$then $N_{+}(-a)=0$ and hence $\mathscr{B}_{+}$can be identified as the norm-closed subcone $\left(\mathscr{B}_{+}, 0\right)$ of $\tilde{\mathscr{B}}_{+}$.

Next we prove 3 -normality of $\tilde{B}_{+}$. If $(a, r) \leqslant(b, s) \leqslant(c, t)$ then

$$
s-r \geqslant N_{+}(a-b), \quad t-s \geqslant N_{+}(b-c) \text {. }
$$

In particular $t \geqslant s \geqslant r$. There are two cases to consider.

Case 1. $s \geqslant 0$. If $s \geqslant 0$ then $|t| \geqslant|s|$. Hence

$$
\begin{aligned}
\|(b, s)\| & =N_{+}(b) \vee N_{+}(-b)+|s| \\
& \leqslant\left(N_{+}(c)+t-s\right) \vee\left(N_{+}(-a)+s-r\right)+|t| \\
& \leqslant\left(N_{+}(c)+|t|\right) \vee\left(N_{+}(-a)+|t|-r\right)+|t| \\
& =N_{+}(c) \vee\left(N_{+}(-a)-r\right)+2|t| \\
& \leqslant\|(c, t)\| \vee\|(a, r)\|+2\|(c, t)\| \\
& \leqslant 3\|(a, r)\| \vee\|(c, t)\| .
\end{aligned}
$$

Case 2. $s \leqslant 0$. If $s \leqslant 0$ then $|r| \geqslant|s|$. Hence

$$
\begin{aligned}
\|(b, s)\| & \leqslant\left(N_{+}(c)+t-s\right) \vee\left(N_{+}(-a)+s-r\right)+|r| \\
& \leqslant\left(N_{+}(c)+t+|r|\right) \vee\left(N_{+}(-a)+|r|\right)+|r| \\
& =\left(N_{+}(c)+t\right) \vee N_{+}(-a)+2|r| \\
& \leqslant\|(c, t)\| \vee\|(a, r)\|+2\|(a, r)\| \\
& \leqslant 3\|(a, r)\| \vee\|(c, t)\| .
\end{aligned}
$$

Since $\tilde{\mathscr{B}}_{+}$is 3 -normal the norm $\|\cdot\|$ and order-norm $\|\cdot\|_{+}$on $\tilde{\mathscr{G}}$ are equivalent, by Proposition 1.2. Thus $\tilde{\mathscr{B}}$ is $\|\cdot\|_{+}$-complete and $\tilde{\mathscr{B}}_{+}$is $\|\cdot\|_{+}$-closed.

Next if $e=(0,1)$ then

$$
e\|(a, t)\|-(a, t)=\left(-a,\|a\|_{+}+|t|-t\right) \in \tilde{\mathscr{B}}_{+}
$$


because $\|a\|_{+}+|t|-t \geqslant N_{+}(a)$. Therefore $e$ is maximal in the unit ball of $\left(\tilde{\mathscr{S}}_{\hat{B}}, \tilde{\mathscr{B}}_{+},\|\cdot\|\right)$, that is, $e$ is an identity of $\left(\mathscr{B}_{,}, \tilde{\mathscr{B}}_{+},\|\cdot\|\right)$. It now follows from Proposition 1.6 that $e$ is an identity of $\left(\tilde{\mathscr{B}}, \tilde{\mathscr{B}}_{+},\|\cdot\|_{+}\right)$where $\|\cdot\|_{+}$denotes the order-norm on $\tilde{\mathcal{B}}$. Moreover this norm is given by

$$
\|(a, t)\|_{+}=\inf \{\lambda \geqslant 0 ;-\lambda e \leqslant(a, t) \leqslant \lambda e\} .
$$

Since $(a, t) \leqslant \lambda e$ is equivalent to $(-a, \lambda-t) \in \tilde{\mathscr{B}}_{+}$one must have

$$
\lambda \geqslant N_{+}(a)+t \text {. }
$$

Similarly $-\lambda e \leqslant(a, t)$ gives $\lambda \geqslant N_{+}(-a)-t$. Therefore the order half-norm and order-norm on $\tilde{\mathscr{B}}$ are given by

$$
N_{+}((a, t))=\left(N_{+}(a)+t\right) \vee 0
$$

and

$$
\|(a, t)\|_{+}=\left(N_{+}(a)+t\right) \vee\left(N_{+}(-a)-t\right) .
$$

This verifies the last statement of the theorem.

ExAmple 2.2. If $\mathscr{B}_{+}=\{0\}$ then $N_{+}(a)=\|a\|$ and and $\|a\|_{+}=\|a\|$. Thus $\tilde{\mathscr{B}}_{+}=\{(a, t) ; t \geqslant\|a\|\}$ and $\|(a, t)\|=\|a\|+|t|=(\|a\|+t) \vee(\|a\|-t)=$ $\|(a, t)\|_{+}$. Therefore the norm and order-norm on $\tilde{\mathscr{B}}$ coincide. Note that $\tilde{\mathscr{B}}_{+}$is non-trivial despite the triviality of $\mathfrak{B}_{+}$.

EXAMPLE 2.3. If $\mathscr{B}$ is the hermitian part of a $C^{*}$-algebra $\mathfrak{A}$, ordered by the positive elements $\mathfrak{A}_{+}$of $\mathfrak{A}$, then the construction of Theorem 2.1 coincides with the addition of an algebraic identity [5]. Since $N_{+}(a)=\left\|a_{+}\right\|$, where $a_{+}$is the positive part of $a$, and since the $C^{*}$-norm and order-norm coincide (Example 1.4) one has the connection

$$
\|(a, t)\|=\left(\left\|a_{+}\right\|+t\right) \vee\left(\left\|a_{-}\right\|-t\right)
$$

between the $C^{*}$-norms on $\tilde{\mathscr{B}}$ and $\mathscr{B}$.

\section{Positive operators}

In this section we examine the extension of operators from the ordered Banach space $\left(\mathscr{B}, \mathscr{B}_{+},\|\cdot\|\right)$ to the space $\left(\tilde{\mathscr{B}}, \tilde{\mathscr{B}}_{+},\|\cdot\|_{+}\right)$with identity $e$ constructed in Theorem 2.1. If $\mathscr{B}_{+}$is normal $\|\cdot\|$ and $\|\cdot\|_{+}$are equivalent norms by Proposition $1.2, \mathscr{B}$ is $\|\cdot\|_{+}$-complete, $\mathscr{B}_{+}$is $\|\cdot\|_{+}$-closed, and each bounded linear operator $A$ on $\left(\mathscr{B}, \mathscr{B}_{+},\|\cdot\|\right)$ defines a bounded operator on the renormed space $\left(\mathscr{B}, \mathscr{B}_{+}\right.$, $\left.\|\cdot\|_{+}\right)$. Therefore we can unambiguously consider the extension of $A$ from $\left(\mathscr{R}, \mathscr{B}_{+},\|\cdot\|\right)$ to $\left(\tilde{\mathscr{B}}, \tilde{\mathscr{B}}_{+},\|\cdot\|_{+}\right)$. 
First we give a characterization of positive operators, that is, operators $A$ with the property that $A \mathscr{B}_{+} \subseteq \mathscr{B}_{+}$.

LemMa 3.1. Let $A$ be a bounded linear operator on the ordered Banach space $\left(\mathscr{B}, \mathscr{B}_{+},\|\cdot\|\right)$. The following conditions are equivalent:

1. $A \mathscr{B}_{+} \subseteq \mathscr{B}_{+}$,

2. $N_{+}(A a) \leqslant\|A\| N_{+}(a), a \in \mathscr{B}$,

3. $N_{+}(A a) \leqslant \alpha N_{+}(a), a \in \mathscr{B}$, for some $\alpha \geqslant\|A\|$.

Proof. $1 \Rightarrow 2$. One has

$$
\begin{aligned}
N_{+}(A a) & =\inf \left\{\|A a+b\| ; b \in \mathscr{B}_{+}\right\} \\
& \leqslant \inf \left\{\|A a+A b\| ; b \in \mathscr{B}_{+}\right\} \\
& \leqslant\|A\| N_{+}(a) .
\end{aligned}
$$

$2 \Rightarrow 3$. This is trivial.

$3 \Rightarrow 1$. If $a \in \mathscr{B}_{+}$then $N_{+}(-a)=0$ and hence $N_{+}(-A a)=0$. But this is equivalent to $A a \in \mathscr{B}_{+}$.

Now suppose that $\mathscr{B}_{+}$is normal and hence the bounded linear operator $A$ on $\mathscr{B}$ is a bounded operator on the renormed space $\left(\mathscr{B},\|\cdot\|_{+}\right)$. The simplest form of extension of $A$ from $\mathscr{B}$ to $\tilde{\mathscr{B}}$ is defined by

$$
A_{\alpha}(a, t)=(A a, \alpha t)
$$

where $\alpha \in \mathbf{R}$. Note that $A_{\alpha}$ is automatically linear and we next examine criteria for it to be positive.

Theorem 3.2. Let $A$ be a bounded linear operator on the ordered Banach space $\left(\mathscr{B}, \mathscr{B}_{+},\|\cdot\|\right)$. Assume $\mathscr{B}_{+}$is normal and consider the extension $A_{\alpha}$ of $A$ to the extended space $\left(\tilde{B}, \tilde{B}_{+},\|\cdot\|_{+}\right)$with identity $e$. If $\alpha \geqslant\|A\|$ the following conditions are equivalent:

1. $A \mathscr{B}_{+} \subseteq \mathscr{B}_{+}$,

2. $A_{\alpha} \tilde{\mathscr{B}}_{+} \subseteq \widetilde{\mathscr{B}}_{+}$,

3. $\left\|A_{\alpha}\right\|=\alpha$,

4. $\left\|A_{\alpha}\right\| \leqslant \alpha$.

Proof. $2 \Rightarrow 1$. If $a \in \mathscr{B}_{+}$then $(a, 0) \in \tilde{\mathscr{B}}_{+}$and $A_{\alpha}(a, 0)=(A a, 0) \subseteq \tilde{\mathscr{B}}_{+}$by assumption. Thus $N_{+}(-A a)=0$ and $A a \in \mathscr{B}_{+}$. 
$1 \Rightarrow 3$. Because $\alpha \geqslant\|A\|$ Condition 1 is equivalent to $N_{+}(A a) \leqslant \alpha N_{+}(a)$ for all $a \in \mathfrak{B}$ by Lemma 3.1. Therefore

$$
\begin{aligned}
N_{+}\left(A_{\alpha}(a, t)\right) & =\left(N_{+}(A a)+\alpha t\right) \vee 0 \\
& \leqslant \alpha\left(N_{+}(a)+t\right) \vee 0 \\
& =\alpha N_{+}((a, t)) .
\end{aligned}
$$

Consequently

$$
\left\|A_{\alpha}(a, t)\right\|_{+} \leqslant \alpha\|(a, t)\|_{+} .
$$

But one also has $\left\|A_{\alpha} e\right\|_{+}=\alpha\|e\|_{+}=\alpha$ and hence $\left\|A_{\alpha}\right\|=\alpha$.

$3 \Rightarrow 4$. This is evident.

To conclude the proof we recall that since $e$ is an identity of $\left(\tilde{\mathscr{B}}, \tilde{\mathscr{B}}_{+},\|\cdot\|_{+}\right)$

$$
\|e-(a, t)\|_{+} \leqslant 1
$$

implies $(a, t) \in \tilde{\mathscr{B}}_{+}$. Conversely, if $(a, t) \in \tilde{\mathscr{B}}_{+}$and $\|(a, t)\|_{+} \leqslant 1$ then $N_{+}(-a)$ $\leqslant t$ and $N_{+}(a)+t \leqslant 1$. Therefore

$$
\|e-(a, t)\|_{+}=\left(N_{+}(-a)+1-t\right) \vee\left(N_{+}(a)-1+t\right) \leqslant 1 .
$$

$4 \Rightarrow 2$. Assume $(a, t) \in \tilde{\mathscr{B}}_{+}$and $\|(a, t)\|_{+} \leqslant 1$. Then setting $B=A_{\alpha} / \alpha$ one has $B e=e$ and hence

$$
\begin{aligned}
\|e-B(a, t)\|_{+} & =\left\|A_{\alpha}(e-(a, t))\right\|_{+} / \alpha \\
& =\left(\left\|A_{\alpha}\right\| / \alpha\right)\|(e-(a, t))\|_{+} \leqslant 1
\end{aligned}
$$

by Condition 4 and the above. Therefore $B(a, t) \in \tilde{\mathscr{B}}_{+}$and consequently $A_{\alpha} \tilde{\mathscr{B}}_{+}$ $\subseteq \tilde{\mathscr{B}}_{+}$.

Remark. The equivalence of Conditions 2 and 3 is an analogue of the $C^{*}$-algebraic result that an operator which leaves the identity fixed is positive if, and only if, it has norm one (see, for example, [5] Corollary 3.2.6).

\section{References}

[1] E. M. Alfsen, Compact convex sets and boundary integrals (Springer-Verlag, 1971).

[2] W. Arendt, P. R. Chernoff and T. Kato, 'A generalization of dissipativity and positive semigroups,' J. Operator Theory 8 (1982), 167-180.

[3] L. Asimov and A. J. Ellis, Convexity theory and its application in functional analysis (Academic Press, 1980).

[4] O. Bratteli, T. Digernes and D. W. Robinson, 'Positive semigroups on ordered Banach spaces,' J. Operator Theory, to appear.

[5] O. Bratteli and D. W. Robinson, Operator algebras and quantum statistical mechanics, Vol. I (Springer-Verlag, 1979). 
[6] J. Grosberg and M. Krein, 'Sur la décomposition des fonctionelles en composantes positives,' $C$. R. Acad. Sci. URSS 25 (1939), 723-726.

[7] R. V. Kadison, 'A representation theory for commutative topological algebras,' Mem. Amer. Math. Soc. No. 7 (1951).

[8] M. Krein, 'Propriétés fondamentales des ensembles coniques normaux dans l'espace de Banach,' C. R. Acad. Sci. URSS 28 (1940), 13-17.

[9] A. L. Peressini, Ordered topological vector spaces (Harper and Rowe, 1967).

[10] H. H. Schaefer, Banach lattices and positive operators (Springer-Verlag, 1974).

\section{Department of Mathematics}

Institute of Advanced Studies

The Australian National University

P.O. Box 4

Canberra, A.C.T. 2600

Australia 